\title{
ELECTROMAGNETIC EFFECTS ON A MOLECULE AT A METAL SURFACE
}

\section{Effects of nonlocality and finite molecular size}

G.W. FORD

Department of Physics, The University of Michigan, Ann Arbor, Michigan 48109, USA

and

W.H. WEBER

Physics Department, Research Staff, Ford Motor Company, Dearborn, Michigan 48121, USA

Received 13 January 1981

The electromagnetic effects on a molecule near a metal surface are considered with the view to understanding the surface-nhanced-Raman-scattering (SERS) effect. The image enhancement effect is calculated including the nonlocal response of the metal and finite molecular size. The effect is much reduced $\left(\times 10^{-5}\right)$ from that for a point molecule above a local metal but can still give a gain $\approx 10^{3}$. The power emitted by a dipole above a smooth surface is also calculated. For an Ag surface the power emitted in the form of photons, surface plasmons, and electron-hole excitations are found to be in the ratio $1: 3: 10^{6}$. The numerical results are calculated using the semi-classical infinite-barrier model of the metal surface with a Lindhard dielectric function modified to take into account finite electron lifetime and core polarization.

\section{Introduction}

The electromagnetic interactions of molecules near a metal surface are of interest in relation to a number of phenomena. Perhaps the most striking is the large enhancement of Raman scattering by molecules adsorbed on certain metal surfaces [1]. Other such phenomena are the surface-induced changes in molecular fluorescence [2], infrared absorption, and vibrational frequencies [3-5]. The Van der Waals forces between molecules and metal surfaces are another such phenomenon [6]. In this paper we discuss the electromagnetic interactions of a molecule at a smooth metal surface. The important effects of surface roughness will be the subject of a subsequent paper.

Although our results are not limited to the Raman scattering application, it is the one which has motivated this work, and we will couch our discussion in terms 
of it. The increase in Raman scattering cross section by adsorbed molecules can be up to six orders of magnitude. A high-reflectivity metal and submicroscopic roughness seem to be important, but the effect is not specific to the molecule. The recent observation of enhanced Raman scattering from molecules on liquid $\mathrm{Hg}$ indicates that resonances associated with roughness features or adatoms may not be essential [7]. The dependence on pump frequency shows no resonant structure, nor does it follow the usual $\omega^{4}$-dependence observed for free molecules. There is apparently a short-range effect requiring direct or nearly direct contact with the metal and a long-range effect which exists for molecules $\sim 10^{2} \AA$ from the surface [8-11]. Any explanation of the surface-enhanced-Raman-scattering (SERS) effect must include these general features.

In section 2 we discuss the enhancement of the effective molecular polarizability by the image fields induced by the metal surface. This image effect was proposed by King et al. [12] and Efrima and Metiu [13], who considered the model of a point dipole above a metallic half-space characterized by a local dielectric constant $\epsilon(\omega)$. Although this model predicts a large effect, it requires a precise spacing of the dipole at distances $1-2 \AA$ from the surface, or for a given spacing exhibits a sharp resonance in pump frequency. At such distances, however, it is clearly necessary to take into account finite molecular size and the nonlocal response of the metal. The modifications due to nonlocality have been discussed previously by us [14] and also by Korzeniewski et al. [15]. In section 2 we show for the first time how both these effects can be taken into account in a calculation of the image effect. For reasonable models of the molecule and the metal we find that the image enhancement, while much reduced, is still significant and, moreover, does not exhibit the resonance structure for a point dipole.

In section 3 we discuss the power dissipation by a dipole near a metal surface. The principal effects are the emission of surface plasmons and the excitation of electron-hole pairs. For a smooth surface these effects do not lead to an increased Raman signal since they correspond to surface waves which dissipate in the metal. Surface roughness can change this dramatically [14]. On the other hand these effects on a smooth surface are important for determining the lifetime of fluorescing molecules, and they have been observed experimentally $[16,17]$.

\section{Image enhancement}

Here we are interested in calculating the induced dipole moment $\mu$ in a molecule situated above a smooth metal surface subject to an applied optical field $E_{0} \mathrm{e}^{-\mathrm{i} \omega t}$. This calculation is particularly simple for the case of a point polarizable dipole and a metal described by a local dielectric constant $\epsilon_{2}(\omega)$. When the distance $d$ of the molecule from the surface is very much less than the optical wavelength it is appropriate to use the quasistatic approximation [18]. The effect of the surface can then 
be represented by an image dipole a distance $d$ below the surface of strength

$\mu_{\mathrm{image}}=\frac{\epsilon_{2}(\omega)-\epsilon_{1}}{\epsilon_{2}(\omega)+\epsilon_{1}}(2 \mu \cdot \hat{z} \hat{z}-\mu)$,

where $\epsilon_{1}$ is the dielectric constant in the medium above the surface, which is taken to be the $x-y$ plane (see fig. 1). The polarizable dipole feels both the applied field and the field of this image,

$\mu=\alpha_{1}\left(E_{0}+E_{\text {image }}\right)$,

where $\alpha_{1}$ is the dipole polarizability and

$E_{\text {image }}=\frac{1}{\epsilon_{1}(2 d)^{3}}\left(3 \mu_{\text {image }} \cdot \hat{z} \hat{z}-\mu_{\text {image }}\right)$.

Solving for $\mu$ we find the familiar result [13]

$$
\begin{aligned}
\mu= & \frac{\alpha_{1}}{1-\left(\alpha_{1} / 4 \epsilon_{1} d^{3}\right)\left[\epsilon_{2}(\omega)-\epsilon_{1}\right] /\left[\epsilon_{2}(\omega)+\epsilon_{1}\right]} \hat{z} \cdot E_{0} \hat{z} \\
& +\frac{\alpha_{1}}{1-\left(\alpha_{1} / 8 \epsilon_{1} d^{3}\right)\left[\epsilon_{2}(\omega)-\epsilon_{1}\right] /\left[\epsilon_{2}(\omega)+\epsilon_{1}\right]}\left(E_{0}-\hat{z} \cdot E_{0} \hat{z}\right) .
\end{aligned}
$$

The image enhancement effect is associated with the near vanishing of the denominators in eq. (4), which typically occurs at distances $d$ of order 1-2 $\AA$. The main purpose of the remainder of this section will be to calculate the induced dipole moment taking into account finite molecular size and the nonlocal response of the metal.

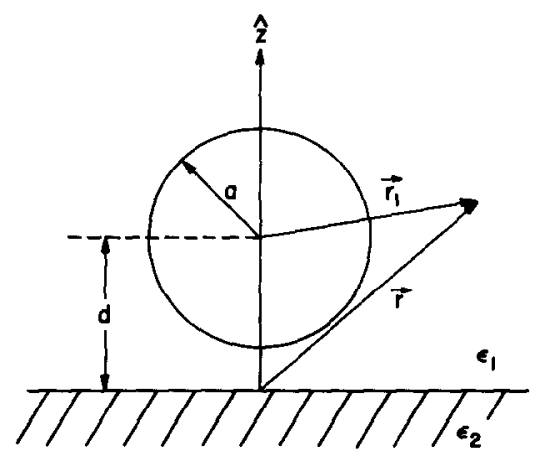

Fig. 1. Schematic drawing of an adsorbed molecule of radius $a$ above a metal half-space. 


\subsection{General formulation}

We consider a finite spherical molecule of radius $a$ centered a distance $d>a$ from a smooth metal surface (fig. 1). The metal surface is completely described by the amplitude reflection coefficients for S- and P-polarized waves. To define these quantities, we first introduce cylindrical coordinates, $\rho=x \hat{x}+y \hat{y}, z$. The S-polarized waves have electric vectors oriented perpendicular to the plane of incidence, so the electric field associated with an incident plane wave of frequency $\omega$ can be written

$\boldsymbol{E}=E_{\mathrm{S}} \mathrm{e}^{\mathrm{i} p \cdot \rho} \hat{z} \times \hat{p}\left[\exp \left(-\mathrm{i} q_{1} z\right)+r_{12}^{\mathrm{S}} \exp \left(\mathrm{i} q_{1} z\right)\right], z>0$,

where $p$ is the projection of the propagation vector on the metal surface, and

$q_{1}=\left(k_{1}^{2}-p^{2}\right)^{1 / 2}, \quad k_{1}^{2} \equiv \epsilon_{1} \omega^{2} / c^{2}, \quad \operatorname{Re}\left\{q_{1}\right\}>0$.

Eq. (5) defines the amplitude reflection coefficient $r_{12}^{\mathrm{S}}(p, \omega)$ for S-polarized waves incident in medium 1 on the surface of medium 2. The corresponding magnetic field is obtained from Faraday's law $[B=-\mathrm{i}(c / \omega)$ curl $E]$,

$\boldsymbol{B}=\frac{c}{\omega} E_{\mathrm{S}} \mathrm{e}^{\mathrm{i} p \cdot \rho}\left[\left(p \hat{z}+q_{1} \hat{p}\right) \exp \left(\mathrm{i} q_{1} z\right)+r_{12}^{\mathrm{S}}\left(p \hat{z}-q_{1} \hat{p}\right) \exp \left(\mathrm{i} q_{1} z\right)\right]$.

The P-polarized waves have magnetic vector oriented perpendicular to the plane of incidence so the magnetic field associated with an incident plane wave can be written

$\boldsymbol{B}=B_{\mathrm{P}} \mathrm{e}^{\mathrm{i} p \cdot \rho} \hat{z} \times \hat{p}\left[\exp \left(-\mathrm{i} q_{1} z\right)+r_{12}^{\mathrm{P}} \exp \left(\mathrm{i} q_{1} z\right)\right], \quad z>0$.

Here $r_{12}^{\mathrm{P}}(p, \omega)$ is the amplitude reflection coefficient for P-polarized waves incident in medium 1 on the surface of medium 2 . The corresponding electric field is given by Ampère's law $\left[E=\mathrm{i}\left(c / \epsilon_{1} \omega\right) \operatorname{curl} \boldsymbol{B}\right]$,

$$
E=-\frac{c}{\omega \epsilon_{1}} B_{\Gamma} \mathrm{e}^{\mathrm{i} \rho \cdot \rho}\left[\left(p \hat{z}+q_{1} \hat{p}\right) \exp \left(-\mathrm{i} q_{1} z\right)+r_{12}^{\mathrm{P}}\left(p \hat{z}-q_{1} \hat{p}\right) \exp \left(\mathrm{i} q_{1} z\right)\right] .
$$

In the consideration of the image-enhancement effect we will be interested in the near zone, distances from the dipole small compared with the wavelength of the incident light. In this case we can use the quasistatic approximation [18], which is equivalent to taking the limit $c \rightarrow \infty\left(q_{1} \rightarrow i p\right)$ in the above equations. In this limit one must keep $c E_{\mathrm{S}}$ and $c B_{\mathrm{P}}$ finite, so the electric field vanishes for $\mathrm{S}$ polarized waves.

In this same quasistatic approximation, the molecule (assumed spherically symmetric) is completely described by its multipole polarizabilities. These quantities are defined in terms of the response of the molecule to an applied multipole field. For the $l$ th multipole the quasistatic electric field outside the molecule is derivable ( $E$ $=-\mathbf{V} \phi)$ from a potential of the form [19]

$\Phi_{l m} \propto\left(-r_{1}^{l}+\frac{\alpha_{1}}{\epsilon_{1} r_{1}^{l+1}}\right) P_{l}^{m}\left(\cos \theta_{1}\right) \exp \left(\mathrm{i} m \phi_{1}\right), \quad r_{1}>a$, 
where $r_{1}$ is the position vector from the center of the molecule, $\theta_{1}$ and $\phi_{1}$ are the polar angles of $\hat{r}_{1}$, and $P_{l}^{m}$ is the associated Legendre polynomial. In (10) the first term is the potential of the applied multipole and the second is the potential due to the induced multipole moment. The coefficient $\alpha_{l}(\omega)$ is the lth multipole polarizability. The radius $a$ is the confining radius of the molecule, the radius outside which the fields are harmonic.

We now address the problem of the molecule above the metal in the presence of a uniform quasistatic electric field. The potential in the region above the metal and outside the molecule can be written [20]

$\Phi=-E_{0} \cdot r+\sum_{l=1}^{\infty} \sum_{m=-l}^{l} B_{l m} r_{1}^{-l-1} P_{l}^{m}\left(\cos \theta_{1}\right) \exp \left(i m \phi_{1}\right)+\int \mathrm{d} p B(p) \mathrm{e}^{\mathrm{i} p \cdot \rho-p z}$,

where (fig. 1)

$r=\rho+z \hat{z}=r_{1}+\mathrm{d} \hat{z}$.

In (11) the first term is the potential of the applied field, the second is that of the field due to the induced multipoles in the molecule, and the last is that of the field reflected from the metal surface. Just outside the molecule the potential must be a superposition of potentials of the form (10). Just above the metal surface the potential must be a superposition of potentials of the form

$\Phi_{p} \propto \mathrm{e}^{\mathrm{i} p \cdot p}\left(\mathrm{e}^{p z}-r_{12}^{p} \mathrm{e}^{-p z}\right)$,

which corresponds to the form (9) for the fields in the quasistatic limit $q_{1}=\mathrm{i} p$. These conditions at the boundaries serve to determine the coefficients in (11).

In applying the boundary conditions we need a pair of crucial formulas. The first is

$\mathrm{e}^{\mathrm{i} p \cdot p-p z}=\mathrm{e}^{-p d} \sum_{l=0}^{\infty} \sum_{m=-t}^{l} \frac{(\mathrm{i})^{m}\left(-p r_{1}\right)^{l}}{(l+m) !} P_{l}^{m}\left(\cos \theta_{1}\right) \exp \left[\mathrm{im}\left(\phi_{1}-\phi\right)\right]$,

where $\phi$ is the azimuthal angle of $\hat{p}$. This formula holds for $z>0$ and serves to express the field reflected from the metal in terms of incident multipole fields at the molecule. The second formula is

$r_{1}^{-l-1} P_{l}^{m}\left(\cos \theta_{1}\right) \exp \left(\mathrm{i} m \phi_{1}\right)=\frac{(-\mathrm{i})^{m}(-)^{l}}{2 \pi(l-m) !} \int \mathrm{d} p p^{l-1} \mathrm{e}^{-p d} \mathrm{e}^{\mathrm{i} p \cdot \rho+p z} \mathrm{e}^{\mathrm{i} m \phi}$.

This formula holds for $z<d$ and serves to express the induced multipole fields from the molecule in terms of plane waves incident on the surface. These formulas are derived in appendix $A$.

Just outside the confining radius of the molecule $r_{1}=a$, we put the expansion 
(14) in the integral in (11), which gives

$$
\begin{aligned}
\Phi= & -\mathrm{d} \hat{z} \cdot \boldsymbol{E}_{0}-r_{1} \boldsymbol{E}_{0} \cdot \hat{r}_{1}+\int \mathrm{d} \boldsymbol{p} B(\boldsymbol{p}) \mathrm{e}^{-\boldsymbol{p d}} \\
& +\sum_{l=1}^{\infty} \sum_{m=-l}^{l}\left[B_{l m^{2}} r_{1}^{l-1}+\frac{\mathrm{i}^{m}}{(l+m) !} \int\right. \\
& \times \mathrm{d} \boldsymbol{p} B(\boldsymbol{p})(-p)^{l} \mathrm{e}^{\left.-p d-\mathrm{i} m \phi_{r_{1}}\right] P_{l}^{m}\left(\cos \theta_{1}\right) \exp \left(\mathrm{i} m \phi_{1}\right) .}
\end{aligned}
$$

Noting that $\left(P_{1}^{0}=\cos \theta, P_{1}^{1}=-2 P_{1}^{-1}=-\sin \theta\right)$

$$
\begin{aligned}
& \left.\boldsymbol{E}_{0} \cdot \hat{r}_{1}=\hat{z} \cdot \boldsymbol{E}_{0} P_{1}\left(\cos \theta_{1}\right)-\frac{1}{2}(\hat{x}-\mathrm{i} \hat{y}) \cdot \boldsymbol{E}_{0} P_{1}^{1}\left(\cos \theta_{1}\right) \exp \left(\mathrm{i} \phi_{1}\right)\right) \\
& \quad+(\hat{x}+\mathrm{i} \hat{y}) \cdot \boldsymbol{E}_{0} P_{1}^{-1}\left(\cos \theta_{1}\right) \exp \left(-\mathrm{i} \phi_{1}\right)
\end{aligned}
$$

and requiring that (16) be a superposition of potentials of the form (10) we see that for $l \geqslant 1$,

$$
\begin{aligned}
& \epsilon_{1}\left(\alpha_{l}\right)^{-1} B_{l m}+\frac{i^{m}}{(l+m) !} \int \mathrm{d} \boldsymbol{p} B(\boldsymbol{p})(-p)^{l} \mathrm{e}^{-p d-\mathrm{i} m \phi} \\
& \quad=\delta_{l, 1}\left[\hat{z} \cdot E_{0} \delta_{m, 0}-\frac{1}{2}(\hat{x}-\mathrm{i} \hat{y}) \cdot \boldsymbol{E}_{0} \delta_{m, 1}+(\hat{x}+\mathrm{i} \hat{y}) \cdot \boldsymbol{E}_{0} \delta_{m,-1}\right] .
\end{aligned}
$$

The $l=0$ terms in (16) simply adjust the potential at the molecule.

Next consider the potential just above the metal surface. There we put the integral expression (15) in the sum in (11) which gives

$\Phi=-E_{0} \cdot r+\int \mathrm{d} \boldsymbol{p} \mathrm{e}^{\mathrm{i} p \cdot \rho}\left[-\sum_{l=1}^{\infty} \sum_{m=-l}^{l} B_{l m} \frac{(-\mathrm{i})^{m}(-p)^{l-1}}{2 \pi(l-m) !} \mathrm{e}^{-p d+\mathrm{i} m \phi} \mathrm{e}^{p z}+B(p) \mathrm{e}^{-p z}\right]$.

Requiring that this be a superposition of potentials of the form (13) gives

$B(p)=r_{12}^{\mathrm{P}} \sum_{l=1}^{\infty} \sum_{m=-l}^{l} B_{l m} \frac{(-\mathrm{i})^{m}(-p)^{l-1}}{2 \pi(l-m) !} \mathrm{e}^{-p d+\mathrm{i} m \phi}$.

There is no inhomogeneous term as in (18) since a uniform field is compatible with the boundary conditions.

Inserting the relation (20) in (18) we get in the second term on the left an integral in which the only dependence on $\phi$, the azimuthal angle of $\boldsymbol{p}$, is a factor $\mathrm{e}^{\mathrm{i}\left(m-m^{\prime}\right) \phi}$, where $m^{\prime}$ is the summation variable in $(20)$. The integral over this azimuthal angle gives a factor of $2 \pi \delta_{m^{\prime}, m}$. The result therefore is an infinite set of coupled inhomogeneous linear equations for the coefficients $B_{l m}$ which we write 
in the form:

$$
\begin{aligned}
& \epsilon_{1}\left(\alpha_{l}\right)^{-1} B_{l m}+\sum_{l^{\prime}=1}^{\infty} \frac{(-1)^{l+l^{\prime}+1}}{(l+m) !\left(l^{\prime}-m\right) !} F_{l l^{\prime}} B_{l^{\prime} m} \\
& \quad=\delta_{l, 1}\left[\hat{z} \cdot E_{0} \delta_{m, 0}-\frac{1}{2}(\hat{x}-\mathrm{i} \hat{y}) \cdot E_{0} \delta_{m, 1}+(\hat{x}+\mathrm{i} \hat{y}) \cdot E_{0} \delta_{m,-1}\right],
\end{aligned}
$$

where

$F_{l l^{\prime}}(\omega, d)=\int_{0}^{\infty} \mathrm{d} p r_{12}^{\mathrm{P}}(p, \omega) p^{l+l^{\prime}} \mathrm{e}^{-2 p d}$.

Since in these equations the coefficients $B_{l m}$ for different values of $m$ are uncoupled (a consequence of our assumption of spherical symmetry for the molecule), we see immediately

$B_{l m}=0, \quad m \neq 0, \pm 1$.

Hence, if we introduce a new set of coefficients $A_{l m}$ defined by

$B_{l, 0}=(-1)^{l+1} \hat{z} \cdot E_{0} A_{l, 0}$,

$B_{l, 1}=(-1)^{l} \frac{1}{2}(\hat{x}-\mathrm{i} \hat{y}) \cdot E_{0} A_{l, 1}$,

$B_{l,-1}=(-1)^{l+1}(\hat{x}+i \hat{y}) \cdot E_{0} A_{l,-1}$,

the coupled equations $(21)$ can be written in the form

$\sum_{l^{\prime}=1}^{\infty} M_{l l^{\prime}}^{m} A_{l^{\prime} m}=\delta_{l, 1}$

where

$M_{l l^{\prime}}^{m}=\frac{\epsilon_{1}}{\alpha_{l}} \delta_{l, l^{\prime}}-\frac{F_{l l^{\prime}}}{(l+m) !\left(l^{\prime}-m\right) !}$.

It is now simple to state the formal solution of these equations using Cramer's rule [21]:

$A_{l m}(\omega, d)=\operatorname{cof}\left(M_{1 l}^{m}\right) / \operatorname{det}\left(M^{m}\right)$.

Here the denominator is the determinant of the infinite matrix $M^{m}$ whose elements are the $M_{\| l^{\prime}}^{m}$ and the numerator is the cofactor of the $1 l$ element in the matrix. Operationally the meaning of (27) is the limit as $n \rightarrow \infty$ of the corresponding ratio formed with the $n$th truncation of $M^{m}$, the $n \times n$ matrix in the upper left hand corner.

The $l=1$ terms in the sum in eq. (11) give the potential $\left(\mu \cdot r / \epsilon_{1} r^{3}\right)$ of the induced dipole moment $\mu$ in the molecule. We thus have, using (24),

$\mu(\omega, d)=\epsilon_{1}\left[A_{1,0} \hat{z} \cdot E_{0} \hat{z}+A_{1,1}\left(E_{0}-\hat{z} \cdot E_{0} \hat{z}\right)\right]$, 
where we have used the fact that, since the matrix $M^{-1}$ is the transpose of the matrix $M^{1}, \quad \mathrm{~A}_{1,-1}=\mathrm{A}_{1,1}$. The procedure for calculating the induced dipole moment is now complete. Given the reflection coefficient $r_{12}^{\mathrm{P}}(p, \omega)$, we construct the integrals (22). Then given the multipole polarizabilities $\alpha_{l}$ we form the matrix $M^{m}$ whose elements are given by (26). The coefficients $A_{1,0}$ and $A_{1,1}$ in (28) are then constructed using (27).

The importance of nonlocal effects can be seen from the integrals $F_{n l^{\prime}}(\omega, d)$ given in (22). The factor $p^{l+l^{\prime}} \mathrm{e}^{-2 p d}$ in the integrand peaks at $p=\left(l+l^{\prime}\right) / 2 d$, which means that $r_{12}^{\mathrm{P}}(p, \omega)$ at these values of $p$ gives the contribution. Thus, if $d$ is on the atomic scale, the dominant wavevector components are on the same scale.

In the special case of a point dipole, the multipole polarizabilities vanish except for $l=1$, where $\alpha_{1}$ is the usual polarizability. In this case the solution of (25) is simple,

$A_{l m}=\frac{\alpha_{1}}{\epsilon_{1}}\left[1-\frac{\alpha_{1} F_{11}}{\epsilon_{1}(1+m) !(1-m) !}\right]^{-1} \delta_{l, 1} \quad$ (point dipole).

For a local dielectric response in the metal, the quasistatic reflection coefficient is $r_{12}^{P}=\frac{\epsilon_{2}(\omega)-\epsilon_{1}}{\epsilon_{2}(\omega)+\epsilon_{1}} \quad$ (local response),

in which case (22) becomes

$F_{l l^{\prime}}=\frac{\left(l+l^{\prime}\right) !}{(2 d)^{l+l^{\prime}+1}} \frac{\epsilon_{2}(\omega)-\epsilon_{1}}{\epsilon_{2}(\omega)+\epsilon_{1}} \quad$ (local response).

Using this in (29) the expression (28) for the induced dipole moment becomes exactly the expression (4) given at the beginning of this section.

\subsection{The reflection coefficients}

In this subsection we consider the calculation of the amplitude reflection coefficients for S- and P-polarized electromagnetic waves incident on the surface of a metal with a nonlocal response. Since we will need the general results later in our discussion of the emission problem we will not make the quasistatic approximation, leaving this to the end where we give results appropriate to the previous subsection.

For an abrupt metal surface it is convenient to formulate the discussion in terms of the surface impedances, defined by

$Z^{\mathrm{P}}(p, \omega)=-\frac{4 \pi}{c}\left\{\frac{\hat{p} \cdot \boldsymbol{E}}{\hat{z} \times \hat{p} \cdot \boldsymbol{B}}\right\}_{\mathrm{inside}} Z^{\mathrm{S}}(p, \omega)=\frac{4 \pi}{c}\left\{\frac{\hat{z} \times \hat{p} \cdot \boldsymbol{E}}{\hat{p} \cdot \boldsymbol{B}}\right\}_{\text {inside }}$,

where the fields are evaluated just inside the metal. The notation here is that of the previous subsection in which $\hat{z}$ is the direction normal to the surface pointing away from the metal, and the variation parallel to the surface is $\mathrm{e}^{\mathrm{i} p \cdot \rho-\omega t}$. Using eqs. (5) through (9) for the fields outside the surface and the standard Maxwell boundary 
conditions of the continuity of the components of $\boldsymbol{E}$ and $\boldsymbol{B}$ parallel to the surface, we obtain the following expressions for the reflection coefficients in terms of the surface impedances:

$r_{12}^{\mathrm{S}}=\frac{\left(c^{2} q_{1} / 4 \pi \omega\right) Z^{\mathrm{S}}-1}{\left(c^{2} q_{1} / 4 \pi \omega\right) Z^{\mathrm{S}}+1}, \quad r_{12}^{\mathrm{P}}=\frac{1-\left(\omega \epsilon_{1} / 4 \pi q_{1}\right) Z^{\mathrm{P}}}{1+\left(\omega \epsilon_{1} / 4 \pi q_{1}\right) Z^{\mathrm{P}}}$.

The calculation of the surface impedance depends upon how one describes the metal. The simplest approach which includes the features of nonlocality is the semiclassical infinite barrier model, which corresponds to the assurnption of specular reflection of the conduction electrons at the metal surface [22.] Here the prescription for computation is simple. One considers the infinite metal with bulk dielectric relation

$\boldsymbol{D}=\epsilon_{\ell}(k, \omega) \hat{k} \cdot \boldsymbol{E} \hat{k}+\epsilon_{\mathbf{t}}(k, \omega)(\boldsymbol{E}-\hat{k} \cdot \boldsymbol{E} \hat{k})$,

where $k$ is the propagation vector and $\epsilon_{\ell}$ and $\epsilon_{\mathrm{t}}$ are the longitudinal and transverse dielectric constants. In this infinite metal one solves the Maxwell equations with a plane current source of the form

$J(r, t)=J \delta(z) \mathrm{e}^{\mathrm{i}(\boldsymbol{p} \cdot \rho-\omega t)}, \quad \hat{z} \cdot \boldsymbol{J}=0$.

The surface impedances are then given by (32) with the fields evaluated just below the plane $z=0$. The result is

$Z^{\mathrm{P}}(p, \omega)=\frac{8 \mathrm{i}}{\omega} \int_{0}^{\infty} \mathrm{d} q \frac{1}{k^{2}}\left(\frac{p^{2}}{\epsilon_{\ell}(k, \omega)}+\frac{q^{2}}{\epsilon_{\mathrm{t}}(k, \omega)-c^{2} k^{2} / \omega^{2}}\right)$,

$Z^{\mathrm{S}}(p, \omega)=\frac{8 \mathrm{i}}{\omega} \int_{0}^{\infty} \mathrm{d} q \frac{1}{\epsilon_{\mathrm{t}}(k, \omega)-c^{2} k^{2} / \omega^{2}}$,

where in these integrals $k^{2}=p^{2}+q^{2}$.

For $\epsilon_{\ell}$ and $\epsilon_{\mathrm{t}}$ we choose the Lindhard form modified for a finite relaxation time [23]:

$\epsilon_{\ell}(k, \omega)=\epsilon_{\mathrm{b}}(\omega)+\frac{3 \omega_{\mathrm{p}}^{2} u^{2} f_{\ell}(z, u)}{(\omega+\mathrm{i} \nu)\left\{\omega+\mathrm{i} \nu\left[f_{\ell}(z, u) / f_{\ell}(z, 0)\right]\right\}}$,

$\epsilon_{\mathrm{t}}(k, \omega)=\epsilon_{\mathrm{b}}(\omega)-\frac{\omega_{\mathrm{p}}^{2} f_{\mathrm{t}}(z, u)}{\omega(\omega+\mathrm{i} \nu)}$,

where

$f_{\ell}(z, u)=\frac{1}{2}+\frac{1}{8 z}\left\{\left[1-(z-u)^{2}\right] \log \frac{z-u+1}{z-u-1}+\left[1-(z+u)^{2}\right] \log \frac{z+u+1}{z+u-1}\right\}$, 
and

$$
\begin{aligned}
& f_{\mathrm{t}}(z, u)=\frac{3}{8}\left(z^{2}+3 u^{2}+1\right) \\
& \quad-\frac{3}{32 z}\left\{\left[1-(z-u)^{2}\right]^{2} \log \frac{z-u+1}{z-u-1}+\left[1-(z+u)^{2}\right]^{2} \log \frac{z+u+1}{z+u-1}\right\} .
\end{aligned}
$$

In (38) and (39)

$z=k / 2 k_{\mathrm{F}}, \quad u=(\omega+\mathrm{i} v) / k v_{\mathrm{F}}$

with $k_{\mathrm{F}}$ the Fermi wave vector, $v_{\mathrm{F}}$ the Fermi velocity, and $\nu=\tau^{-1}$ the collision frequency of the conduction electrons. Also $\omega_{p}=\left(4 \pi n e^{2} / m\right)^{1 / 2}$ is the plasma frequency and $\epsilon_{\mathrm{b}}(\omega)$ is a phenomenological term representing the (local) bound electron response. We should emphasize that the band structure of the noble metals is much more complicated than is implied by our simple free-electron picture [24]. Nevertheless, this modified Lindhard form of the dielectric relation is a reasonable approximation which includes in a consistent manner the excitation spectrum of the conduction electrons.

The quasistatic approximation for $r_{12}^{\mathrm{P}}$ is obtained by taking the limit $c \rightarrow \infty$ in (36). Then from (33) we can write

$$
r_{12}^{\mathrm{P}}(p, \omega)=\frac{1-\frac{2}{\pi} \epsilon_{1} p \int_{0}^{\infty} \mathrm{d} q \frac{1}{k^{2} \epsilon_{\ell}(k, \omega)}}{1+\frac{2}{\pi} \epsilon_{1} p \int_{0}^{\infty} \mathrm{d} q \frac{1}{k^{2} \epsilon_{\ell}(k, \omega)}} \quad \text { (quasistatic) . }
$$

\subsection{Multipole polarizabilities}

The multipole polarizabilities are defined via (10) in terms of the (linear) response of the molecule to an applied multipole field. They are the generalization of the familiar dipole polarizability defined in terms of the response to an applied uniform field. Here we discuss the calculation of these quantities for various one-electron systems. The purpose is to gain insight into the structure of multipole polarizabilities for real molecules and to obtain for special models explicit formulas useful for computation.

For a system with a spherically symmetric ground state the following formula is the direct generalization of the well-known Kramers-Heisenberg dispersion formula for the polarizability [25]

$\alpha_{\ell}=e^{2} \sum_{i}\left|\left\langle\Psi_{i}, r^{l} P_{l}(\cos \theta) \Psi_{0}\right\rangle\right|^{2} \times\left(\frac{1}{E_{i}-E_{0}-\hbar \omega}+\frac{1}{E_{i}-E_{0}+\hbar \omega}\right)$. 
Here the sum is over all intermediate states $\Psi_{i}$, and $\Psi_{0}$ is the ground state. In fact, like the Kramers-Heisenberg formula, this formula is not very useful since, except for the harmonic oscillator, the sum does not converge rapidly. On the other hand, we do see from this formula that when the frequency of the exciting radiation is well below the excitation frequency for the lowest excited state, it can be neglected and the polarizability becomes essentially the static polarizability. For simplicity, in the following discussion we consider the static polarizabilities.

A more useful approach than the Kramers-Heisenberg formula is to solve explicitly for the perturbed wave function [26]. Consider the perturbed Hamiltonian

$H=H_{0}+e r^{l} P_{l}(\cos \theta)$,

where the second term is the perturbing potential of the applied multipole field and

$H_{0}=-\left(\hbar^{2} / 2 m\right) \nabla^{2}+V(r)$

is the unperturbed Hamiltonian. The unperturbed ground state is an eigenstate of $H_{0}$ which we assume is spherically symmetric.

$H_{0} \Psi_{0}=E_{0} \Psi_{0}, \quad \Psi_{0}=\Psi_{0}(r)$.

The perturbed eigenvalue problem is

$\left(H_{0}+e r^{l} P_{l}\right) \Psi=E \Psi$.

Expanding

$\Psi=\Psi_{0}+\Psi^{(1)}+\ldots, \quad E=E_{0}+E^{(1)}+\ldots$,

we find for the first order equation

$\left(H_{0}-E_{0}\right) \Psi^{(1)}=-\operatorname{er}^{i} P_{l} \Psi_{0}+E^{(1)} \Psi_{0}$.

The first order energy is

$E^{(1)}=\int \mathrm{d} r \Psi_{0}^{*}(r) e r^{l} P_{l}(\cos \theta) \Psi_{0}(r)=0$,

because of the spherical symmetry of the ground state. Hence, putting

$\Psi^{(1)}(r)=R_{l}(r) P_{l}(\cos \theta)$

and using (46), the first order equation (50) can be written

$\left[\frac{\hbar^{2}}{2 m}\left(-\frac{1}{r^{2}} \frac{\mathrm{d}}{\mathrm{d} r} r^{2} \frac{\mathrm{d}}{\mathrm{d} r} \frac{l(l+1)}{r^{2}}\right)+V(r)-E_{0}\right] R_{l}=-e r^{l} \Psi_{0}(r)$.

This is an inhomogeneous differential equation for the perturbed radial wave function $R_{7}$.

Having solved the equation for $R_{l}$ we form the induced charge density

$\rho(r)=-e\left(|\Psi|^{2}-\left|\Psi_{0}\right|^{2}\right)=-e\left(\Psi_{0}^{*} R_{l}+\Psi_{0} R_{l}^{*}\right) P_{l}(\cos \theta)+\ldots$. 
The associated electrostatic potential in a medium of dielectric constant $\epsilon_{1}$ is

$$
\begin{aligned}
& \Phi(r)=\int \mathrm{d} r^{\prime} \frac{\rho\left(r^{\prime}\right)}{\epsilon_{1}\left|r-r^{\prime}\right|} \\
& \sim \frac{1}{\epsilon_{1}} \sum_{l=0}^{\infty} \frac{1}{r^{l+1}} \int \mathrm{d} r^{\prime} r^{\prime l} P_{l}\left(\hat{r} \cdot \hat{r}^{\prime}\right) \rho\left(r^{\prime}\right),
\end{aligned}
$$

where we have used the well-known expansion of $\left|\boldsymbol{r}-\boldsymbol{r}^{\prime}\right|^{-1}$ [27]. Using the addition theorem for spherical harmonics

$$
\begin{aligned}
& P_{l}\left(\hat{r} \cdot \hat{r}^{\prime}\right)=P_{l}(\cos \theta) P_{l}\left(\cos \theta^{\prime}\right) \\
& \quad+2 \sum_{m=1}^{l} \frac{(l-m) !}{(l+m) !} P_{l}^{m}\left(\cos \theta^{\prime}\right) P_{l}^{m}\left(\cos \theta^{\prime}\right) \cos m\left(\phi-\phi^{\prime}\right),
\end{aligned}
$$

the form (54) for $\rho$, and the orthogonality of the spherical harmonics [27], we find $\Phi(r) \sim\left(\alpha_{l} / \epsilon_{1} r^{l+1}\right) P_{l}(\cos \theta)$,

where comparing with (10)

$\alpha_{l}=-\frac{4 \pi e}{2 l+1} \int_{0}^{\infty} \mathrm{d} r r^{l+2}\left[\Psi_{0}^{*}(r) R_{l}(r)+\Psi_{0}(r) R_{l}(r)^{*}\right]$

is the multipole polarizability.

For the harmonic oscillator,

$V(r)=\frac{1}{2} m \omega_{0}^{2} r^{2}$,

$\Psi_{0}=\left[m \omega_{0} / \pi \hbar\right]^{3 / 4} \exp \left(-m \omega_{0} r^{2} / 2 \hbar\right)$.

The solution of (53) is

$R_{l}(r)=-\left(e / \hbar \omega_{0}\right) r^{l} \Psi_{0}(r)$.

Putting this in (58) we find

$$
\begin{aligned}
\alpha_{l} & =\frac{8 \pi e^{2}}{l(2 l+1) \hbar \omega_{0}}\left(\frac{m \omega_{0}}{\pi \hbar}\right)^{3 / 2} \int_{0}^{\infty} \mathrm{d} r r^{2 l+2} \exp \left(-m \omega_{0} r^{2} / \hbar\right) \\
& =\frac{e^{2}}{m \omega_{0}^{2}}\left(\frac{\hbar}{2 m \omega_{0}}\right)^{l-1} \frac{(2 l-1) ! !}{l},
\end{aligned}
$$

where $(2 l-1) ! !=1 \cdot 3 \cdot 5 \cdots(2 l-1)$. For $l=1$ this is the familiar result for the polarizability of the harmonic oscillator. For higher $l$ it is interesting that the $\alpha_{l}$ are quantum mechanical, they vanish for the classical oscillator. It is also important to note that $\alpha_{l}$ grows rapidly with $l$; the harmonic oscillator is very "soft". 
Another model for which the solution of (53) is simple is the infinite spherical well,

$V(r)=\left\{\begin{array}{ll}0, & 0<r<a \\ \infty, & a<r\end{array}\right.$.

Here

$\Psi_{0}(r)=\left(\frac{\pi}{2 a^{3}}\right)^{1 / 2} j_{0}\left(\frac{\pi r}{a}\right)=\frac{1}{(2 \pi a)^{1 / 2}} \frac{\sin (\pi r / a)}{r}$,

and the solution of (53) is

$R_{l}(r)=-\frac{1}{l+1} \frac{m e}{\hbar^{2}}\left(\frac{a}{2 \pi^{3}}\right)^{1 / 2}\left[r^{l} \cos (\pi r / a)+a^{l} j_{l}(\pi r / a) / j_{l}(\pi)\right]$,

where $j_{l}$ is the spherical Bessel function [28]. Inserting this in (58) we find

$\alpha_{l}=\frac{4 a^{2 l+2}}{(l+1)(2 l+1) \pi^{l+3} a_{0}} g(l)$

where

$g(l)=\int_{0}^{\pi} \mathrm{d} y\left[y^{2 l+1} \sin y \cos y+\pi^{l} y^{l+1} \sin y j_{l}(y) / j_{l}(\pi)\right]$

and $a_{0}=\hbar^{2} / m e^{2}$ is the Bohr radius. Although the integrals in (67) can be performed explicitly the results are best displayed graphically.

A third simple model which we consider is that of a uniform dielectric sphere of radius $a$, for which the $\alpha_{l}$ are given by

$\alpha_{l}=\frac{\epsilon_{1} l\left(\epsilon_{3}-\epsilon_{1}\right)}{l \epsilon_{3}+(l+1) \epsilon_{1}} a^{2 l+1}$,

where $\epsilon_{3}$ is the dielectric constant of the sphere,;

Each of these three models has certain parameters which must be specified. Since we have in mind applying them to a specific molecule, we will require $\alpha_{1}$ to be the same for each model. This choice fixes $e^{2} / m \omega_{0}^{2}$ for the harmonic oscillator, it fixes the ratio $a^{4} / a_{0}$ for the spherical well, and it relates $\epsilon_{3}$ to $a$ and $\epsilon_{1}$ for the dielectric sphere. The results for these models can now be summarized:

$$
\alpha_{l}=\alpha_{1} \times \begin{cases}\frac{a_{c}^{2 l-2}(2 l-1) ! !}{l 6^{l-1}}, & \text { harmonic oscillator }, \\ \frac{4 a^{2 l-2} g(l)}{(l+1)(2 l+1) \pi^{2 l-1}\left(\pi^{2} / 3+1 / 4\right)}, & \text { spherical well }, \\ \frac{3 l a^{2 l-2}}{(2 l+1)+(l-1) \alpha_{1} / \epsilon_{1} a^{3}}, & \text { dielectric sphere, }\end{cases}
$$


where for the harmonic oscillator we have introduced the classical turning point $a_{\mathrm{c}}$ $=\left(3 \hbar / m \omega_{0}\right)^{1 / 2}$ and for the spherical well we have used the fact that $g(1)=\pi^{3} / 2+$ $3 \pi / 8$. Calculated results for the normalized multipole polarizabilities $\alpha_{l} / \alpha_{1} a^{2 l-2}$ are shown in fig. 2 . Note that we must specify $a / a_{\mathrm{c}}$ for the harmonic oscillator and $\alpha_{1} /$ $\epsilon_{1} a^{3}$ for the dielectric sphere. The normalized multipole polarizabilities for the spherical well decrease rapidly as $l$ increases and vanish as $l \rightarrow \infty$. Its response is most "point-like". For the harmonic oscillator they always diverge as $l \rightarrow \infty$, but the detailed dependence changes with the $a / a_{\mathrm{c}}$ ratio. For the dielectric sphere they are always constrained between 1 and $3 /\left(2+\alpha_{1} / \epsilon_{1} a^{3}\right)$, i.e., the $l=1$ and $l=\infty$ limits.

\subsection{Raman gain for a smooth surface}

The picture one has of the Raman scattering process for an isolated molecule is the following. The molecule is driven by an external field at the pump frequency $\omega_{0}$, which gives rise to an induced dipole moment $\mu\left(\omega_{0}\right)$. An internal vibrational motion, of frequency $\omega_{\mathrm{V}}$, modulates the dipole polarizability of the molecule,

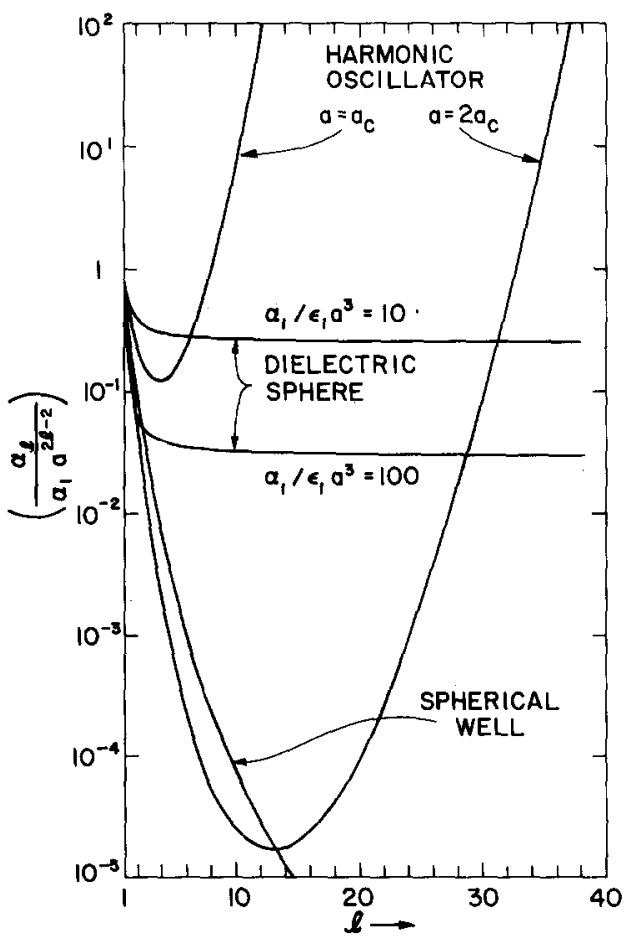

Fig. 2. Multipole polarizabilities as a function of $l$, the multipole order, as given by eq. (69) for three molecular models. 
giving rise to a small induced moment,

$\mu\left(\omega_{R}\right)=c_{R} \mu\left(\omega_{0}\right)$,

which radiates at the Raman frequency $\omega_{R}=\omega_{0} \pm \omega_{V}$. Here $c_{R}$ is a small dimensionless constant characteristic of the internal structure of the molecule. In general $c_{R}$ is a tensor but for simplicity, and in keeping with our previous assumption of a spherical molecule, we take it to be a scalar. For a point-dipole molecule the extension of our discussion to include tensor polarizabilities is only a matter of notation.

The results of section 2.1 . are readily adapted to calculate the Raman scattering by a molecule adsorbed on a smooth surface. The induced moment at the pump frequency is given by $(28)$,

$\mu\left(\omega_{0}\right)=\epsilon_{1}\left[A_{1,0}\left(\omega_{0}, d\right) E_{\perp}+A_{1,1}\left(\omega_{0}, d\right) E_{\|}\right]$,

where $E_{\perp}$ and $E_{\|}$are, respectively, the components of the pump field perpendicular and parallel to the surface. We see from (70) that the induced moment at the Raman frequency is the same as that produced by an external field $E_{0}=c_{\mathrm{R}} \mu\left(\omega_{0}\right) /$ $\alpha_{1}\left(\omega_{R}\right)$ at the Raman frequency. Thus, in the presence of the surface,

$\mu\left(\omega_{\mathrm{R}}\right)=\frac{\epsilon_{1} c_{\mathrm{R}}}{\alpha_{1}\left(\omega_{\mathrm{R}}\right)}\left[A_{1,0}\left(\omega_{\mathrm{R}}, d\right) \mu_{\perp}\left(\omega_{0}\right)+A_{1,1}\left(\omega_{\mathrm{R}}, d\right) \mu_{\|}\left(\omega_{0}\right)\right]$

Using (71) this becomes

$\mu\left(\omega_{\mathrm{R}}\right)=\frac{\epsilon_{1}^{2} c_{\mathrm{R}}}{\alpha_{1}\left(\omega_{\mathrm{R}}\right)}\left[A_{1,0}\left(\omega_{\mathrm{R}}, d\right) A_{1,0}\left(\omega_{0}, d\right) E_{1}+A_{1,1}\left(\omega_{\mathrm{R}}, d\right) A_{1,1}\left(\omega_{0}, d\right) E_{\|}\right]$

In obtaining this result we have assumed that the constant $c_{R}$, being characteristic of the internal structure of the molecule, is unchanged by the presence of the surface.

Since in general the radiated power is proportional to the square of the induced moment, we identify the image enhancement Raman gain $G_{I}$ as the square of the ratio of the magnitude of the induced moment (73) in the presence of the surface to that far from the surface $(d=\infty)$. From (22), (25), and (26) it is easy to see that

$A_{1, \mathrm{~m}}(\omega, \infty)=\alpha_{1}(\omega) / \epsilon_{1}$

and, therefore,

$$
\begin{aligned}
G_{\mathrm{I}} & =\frac{\epsilon_{1}^{4}}{\left|\alpha_{1}\left(\omega_{\mathrm{R}}\right) \alpha_{1}\left(\omega_{0}\right)\right|^{2}}\left[\left|A_{1,0}\left(\omega_{\mathrm{R}}, d\right) A_{1,0}\left(\omega_{0}, d\right)\right|^{2} \frac{\left|E_{1}\right|^{2}}{|\boldsymbol{E}|^{2}}\right. \\
& \left.+\left|A_{1,1}\left(\omega_{\mathrm{R}}, d\right) A_{1,1}\left(\omega_{0}, d\right)\right|^{2} \frac{\left|E_{\|}\right|^{2}}{|\boldsymbol{E}|^{2}}\right]
\end{aligned}
$$

where $E=E_{\|}+E_{1}$. 
We should emphasize that this arbitrarily defined image enhancement gain does not include the effects of the surface on the process of radiation at the Raman frequency, nor does it include the modification of the incident pump field by the surface. The radiation process will be considered in section 3 . The modification of the pump field near the surface $(z \approx 0)$ can be seen from $(5)$ and $(9)$, which can be written

$E_{1}=\left(1+r_{12}^{\mathrm{P}}\right) \hat{z} \cdot E_{\mathrm{p}} \hat{z}, \quad E_{\|}=\left(1-r_{12}^{\mathrm{p}}\right)\left(E_{\mathrm{P}}-\hat{z} \cdot E_{\mathrm{P}} \hat{z}\right)+\left(1+r_{12}^{\mathrm{S}}\right) E_{\mathrm{S}}$,

where $E_{\mathrm{p}}$ and $E_{\mathrm{S}}$ are, respectively, the $\mathrm{P}$ and $\mathrm{S}$ components of the incident fields evaluated at the surface. For these optical fields the reflection coefficients can be evaluated treating the metal response as local;

$r_{12}^{\mathrm{p}}=\frac{\epsilon_{2} q_{1}-\epsilon_{1} q_{2}}{\epsilon_{2} q_{1}+\epsilon_{1} q_{2}}, \quad r_{12}^{\mathrm{s}}=\frac{q_{1}-q_{2}}{q_{1}+q_{2}} \quad$ (local)

where $q_{1}$ is given by $(6)$ and $q_{2}=\left(\epsilon_{2} \omega^{2} / c^{2}-p^{2}\right)^{1 / 2}$. For nominal angles of inci. dence, the effects of the surface are to increase $E_{1}$ and decrease $E_{\| l}$, generally by factors less than 2 .

We conclude this section with a remark about the effect of surface roughness on the image enhancement effect. As we have seen in section 2.1, the effect for adsorbed molecules is localized in the sense that it involves primarily wavevector com. ponents of the metal response which are on the atomic scale. If we assume that molecules adsorbed at steps or onto adatoms are not important, an adsorbed molecule will find itself on a surface which is planar in its neighborhood. Thus we expect that the image enhancement effect will be unchanged except possibly for varying orientations of the local surface relative to the mean surface.

\subsection{Numerical results}

For the purpose of obtaining numerical results we have chosen the metal parameters to correspond to those of $\mathrm{Ag}$. The optical $\epsilon_{2}(\omega)$ corresponds to the local (long wavelength) limit of (38) or (39),

$\epsilon_{2}(\omega)=\epsilon_{\mathrm{b}}(\omega)-\omega_{\mathrm{p}}^{2} / \omega(\omega+i \nu)$

We have fitted the optical data in the region $450-700 \mathrm{~nm}$ to obtain $h \omega_{p}=9.33 \mathrm{eV}$, $h \nu=0.058 \mathrm{eV}$, and $\epsilon_{\mathrm{b}}=3.6[29]$. The remaining parameters in the Lindhard dielectric relations (38) and (39) were determined using one electron per atom and the free electron mass, giving $k_{\mathrm{F}}=\left(3 n \pi^{2}\right)^{1 / 3}=1.2 \times 10^{8} \mathrm{~cm}^{-1}$ and $v_{\mathrm{F}}=\hbar k_{\mathrm{F}} / \mathrm{m}=1.4 \times$ $10^{8} \mathrm{~cm} / \mathrm{s}$. Note that we have fitted $\epsilon_{\mathrm{b}}(\omega)$ with a constant and we have treated the parameters $\omega_{\mathrm{p}}, k_{\mathrm{F}}$, and $v_{\mathrm{F}}$ as independent, while in the free electron model they are related.

In fig. 3 we show the image enhancement Raman gain $G_{I}$ versus $d$ for the case of a point dipole molecule with a normal electric field and either a local or nonlocal metal response. The molecular polarizability was chosen to be $\alpha_{1}=11.3 \AA^{3}$, which 


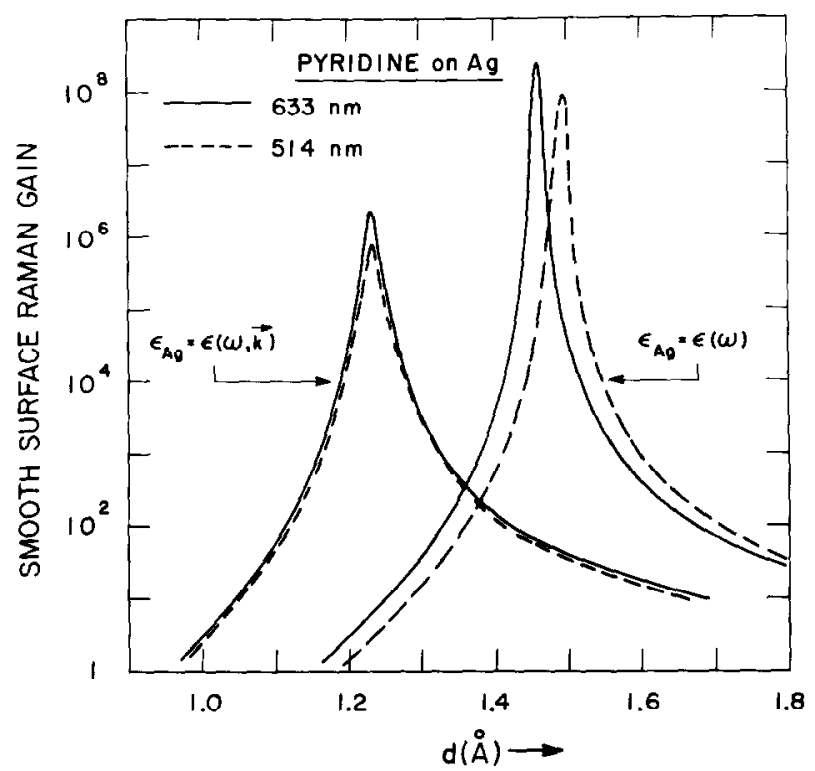

Fig. 3. Image enhancement gain $G_{\mathrm{I}}$ versus $d$ for a point dipole with electric field normal to a local $\left[\epsilon_{\mathrm{Ag}}=\epsilon(\omega)\right]$ and a nonlocal $\left[\epsilon_{\mathrm{Ag}}=\epsilon(\omega, k)\right]$ metal surface. The results for 2 pump frequencies are shown and we chose $\alpha_{1}=11.3 \mathrm{~A}^{3}$ and $\omega_{\mathrm{V}}=1000 \mathrm{~cm}^{-1}$, corresponding to pyridine.

corresponds to pyridine adsorbed in an upright orientation [30]. The large peaks in the gain arise from the vanishing of the real part of the denominator in $A_{1,0}(\omega, d)$ given by (29). That is, a peak will occur when

$\operatorname{Re}\left\{F_{11}\right\}=\epsilon_{1} / \alpha_{1}$.

In practice the Raman shifts are small enough that $F_{11}\left(\omega_{0}, d\right) \approx F_{11}\left(\omega_{\mathrm{R}}, d\right)$. The relation (79) gives therefore the distance for maximum gain, and this gain is given by

$$
\left(G_{I}\right)_{\max } \approx\left[\operatorname{Re}\left\{F_{11}\right\} / \operatorname{Im}\left\{F_{11}\right\}\right]^{4} \quad \text { (point dipole). }
$$

The main effects of nonlocality are a reduction of this maximum gain by two orders of magnitude, a small shift in the distance for maximum gain, and a weakened dependence on the pump frequency. These arise from two distinct consequences of the nonlocal response of the metal. The first is that the inability of the conduction electrons to respond to the large wavevector components of the dipole field (screening) reduces the real part of $F_{11}$ and, hence, reduces the distance for maximum gain. This is in qualitative agreement with a static Thomas-Fermi calculation by Antoniewicz [31], but the reduction is substantially less in our calculation. This is due to the presence of the local interband term $\epsilon_{\mathrm{b}}$, which is un- 
screened. The second consequence of nonlocality is that the excitation of electronhole pairs by the large wavevector $\left(k \approx k_{\mathrm{F}}\right)$ components of the dipole field increases the imaginary part of $F_{11}$ and, hence, decreases the maximum gain. This effect is not present in a static calculation.

The effects of nonlocality on the image gain, while significant, do not change the essential features of a maximum gain of several orders of magnitude over a very narrow range of distance. As we see from fig. 3 , the maximum gain is $\approx 10^{6}$ and occurs over a range of a few hundredths of an $\AA$. While the peak gain is adequate to explain the observed SERS, it is difficult to conceive that typical molecules would be spaced so precisely. But the size of real molecules is comparable to this spacing for maximum gain and on such a scale a point dipole is not an accurate model.

In fig. 4 we compare the image gain curves for two different molecular models with finite size, the infinite spherical well and the dielectric sphere. As we indicated in section 3.3 the dielectric sphere model is intermediate between the "soft" harmonic oscillator and the "hard" infinite spherical well and, we feel, is a reasonable model for real molecules. Both models show that for a small sphere $(\$ 1 \AA)$ the point dipole is a good approximation. This is also essentially true for all sizes with

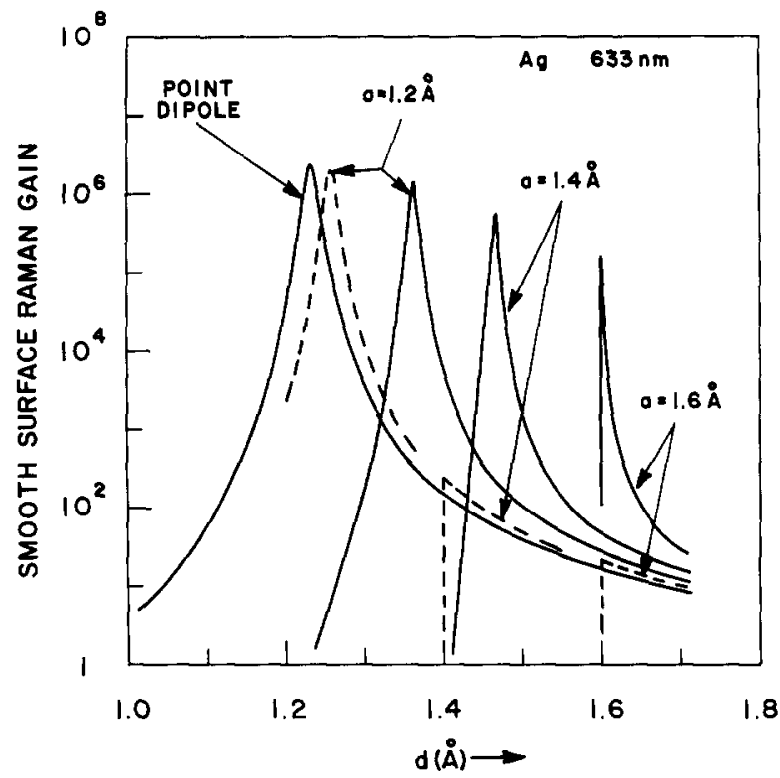

Fig. 4. Image enhancement gain $G_{\mathrm{I}}$ for finite-sized molecules above a nonlocal $\mathrm{Ag}$ metal surface. The molecular radius is a and the pump wavelength is $633 \mathrm{~nm}$. The solid curves correspond to the dielectric sphere model, the dashed curves to the infinite spherical well model. In each case the dipole polarizability is constrained to be $\alpha_{1}=11.3 \AA^{3}$. Both models reduce to a point dipole for small a. 
the infinite spherical well model, which behaves like a point dipole inside an impenetrable shell. For the dielectric sphere model, however, the larger spheres show a dramatic change. The position of maximum gain is pushed out and occurs slightly outside the sphere radius, i.e., the sphere is nearly touching. The peak gain is much reduced and the gain curve is substantially narrowed. Thus while the model implies a reduction in gain it is striking that the peak gain occurs when the molecule rests on the surface, suggesting that the precise placement is not a problem for real molecules. Another effect which occurs for real molecules is the vibrational motion in the adsorption potential. Typical measured vibrational frequencies for this motion are $\omega_{\mathrm{V}} \approx 10^{2} \mathrm{~cm}^{-1}$ and choosing $M \approx 10^{2} \mathrm{amu}$ the amplitude of this motion at room temperature should be

$\left[\left(\hbar / 2 M \omega_{\mathrm{V}}\right) \operatorname{coth}\left(\hbar \omega_{\mathrm{V}} / 2 k T\right)\right]^{1 / 2} \approx 0.1 \AA$.

Averaging the gain curve for a dielectric sphere model with radius $a=1.8 \AA$ over this zero-point motion, which amounts to multiplying the peak gain by the ratio of the width of the gain peak to the amplitude of the zero-point motion, we get an image enhancement Raman gain of $\approx 10^{3}$. In view of the simplicity of the models for which we have been able to compute, we should view this value of the image enhancement Raman gain as at best an order of magnitude estimate. Nevertheless we feel confident that for real molecules the image enhancement gain is present but much too small by itself to explain the SERS experiments.

As pointed out by Efrima and Metiu [13], the image enhancement effect can be viewed as a resonance Raman effect in which the metal produces an apparent downward shift of the natural electronic frequency of the molecule into resonance. From the results shown in fig. 3 one sees that if for a point dipole at a fixed distance above a local metal the gain is plotted versus pump frequency then a narrow peak occurs, analogous to that in resonant Raman scattering. For a point dipole above a nonlocal metal, again as indicated in fig. 3 , the distance for maximum gain is much less sensitive to frequency, so a corresponding frequency plot would be much broader. In either case, although the vibrational motion of the molecule against the surface will broaden the peak, the molecule must be precisely positioned to see any significant gain. For a finite sized molecule our calculations indicate that positioning will be automatic and, therefore, the gain will be only slowly varying with frequency, with no resonant structure. Nevertheless the response corresponds to a strongly coupled motion of the molecule with the conduction electrons in the metal, and in this sense may be termed resonant.

We conclude with a remark concerning the image gain in metals other than Ag. One might think since the gain given by ( 80$)$ is inversely proportional to the fourth power of $\operatorname{Im}\left\{F_{11}\right\}$, which in a local calculation is proportional to the electron scattering rate $\nu$, that free electron metals which have a relatively large $\nu$, such as Al or $\mathrm{Hg}$, would yield a much smaller image gain. This, however, may not be the case since our nonlocal calculation shows that $\operatorname{Im}\left\{F_{11}\right\}$ is determined by electron-hole excitations and is essentially independent of $\nu$. On the other hand metals with inter- 
band absorption, such as $\mathrm{Au}$ or $\mathrm{Cu}$ above $\hbar \omega \approx 2 \mathrm{eV}$, would have a large $\operatorname{Im}\left\{F_{11}\right\}$ arising from a corresponding imaginary part in the local interband term $\epsilon_{\mathrm{b}}$, and would show a much smaller gain. Thus the key property of the metal leading to large image gain is the absence of interband absorption, not small electron scattering rate.

\section{Radiation}

Here we consider the problem of the emission of radiation by a molecule above a metal surface. Since the wavelength of visible radiation is much larger than typical molecular sizes we are justified in most instances in treating the radiating molecule as a point dipole. We therefore treat the problem of an oscillating point dipole above a metal surface, extending the classical Sommerfeld treatment [32] to include the effects of a nonlocal dielectric relation. We should also remark that the results of this section apply to molecular fluorescence as well as to SERS $[2,16,17$, 33].

We consider an oscillating point dipole $\mu(\omega) \mathrm{e}^{-\mathrm{i} \omega t}$ situated a distance $d$ above a metal surface (fig. 1). The time averaged power dissipated by the dipole at frequency $\omega$ is

$P_{\text {tot }}(\omega)=-\frac{1}{2} \omega \operatorname{Im}\left\{\mu^{*}(\omega) \cdot E(\omega)\right\}$,

where $E(\omega)$ is the electric field evaluated at the dipole. To find the electric field we must solve the Maxwell equations in the region above the metal,

$\nabla \times E-i \frac{\omega}{c} B=0$,

$\nabla \times B+\mathrm{i} \epsilon_{1} \frac{\omega}{c} E=-\mathrm{i} 4 \pi \frac{\omega}{c} \mu \delta(\rho) \delta(z-d)$,

together with the boundary conditions at the surface of the metal, In this region the field may be written as a superposition of the field of the dipole in an infinite medium plus the reflected field from the metal,

$E=E_{\text {dipole }}+E_{\text {reflected }}$.

The dipole field is calculated in appendix B, while the reflected field must be a superposition of upward propagating P-and S-polarized waves:

$\boldsymbol{E}_{\text {reflected }}=\int \mathrm{d} \boldsymbol{p} \mathrm{e}^{\mathrm{i} p \cdot \rho}\left[A_{\mathrm{P}}\left(p \hat{z}-q_{1} \hat{p}\right)+A_{\mathrm{S}} \hat{z} \times \hat{p}\right] \exp \left(\mathrm{i} q_{1} z\right)$.

To determine the coefficients $A_{\mathrm{P}}(p)$ and $A_{\mathrm{S}}(p)$ we consider the region $0<z<d$, above the metal but below the dipole. There the dipole field, given in (B.8), can be 
expressed as a superposition of downward propagating P- and S-polarized waves:

$$
\begin{aligned}
& E_{\text {dipole }}=\frac{\mathrm{i}}{2 \pi \epsilon_{1}} \int \mathrm{d} p \frac{1}{q_{1}} \mathrm{e}^{\mathrm{i} p \cdot \rho} \\
& \quad \times\left[\left(p \hat{z}+q_{1} \hat{p}\right) \cdot \mu\left(p \hat{z}+q_{1} \hat{p}\right)+k_{1}^{2} \hat{z} \times \hat{p} \cdot \mu \hat{z} \times \hat{p}\right] \exp \left[-\mathrm{i} q_{1}(z-d)\right] .
\end{aligned}
$$

[The quantity in square brackets is just $-k_{1} \times\left(k_{1} \times \mu\right)$.] But in this region the field (84) must be a superposition of S-polarized fields of the form (5) and P-polarized fields of the form (9). Hence, identifying the incident fields in these expressions with the corresponding fields in the integrand of (86), we find

$A_{\mathrm{P}}=\frac{\mathrm{i}}{2 \pi \epsilon_{1} q_{1}}\left(p z+q_{1} \hat{p}\right) \cdot \mu r_{12}^{\mathrm{P}} \exp \left(\mathrm{i} q_{1} d\right)$

$A_{\mathrm{S}}=\frac{\mathrm{i}}{2 \pi \epsilon_{1} q_{1}} k_{1}^{2} \hat{z} \times \hat{p} \cdot \mu r_{12}^{\mathrm{S}} \exp \left(\mathrm{i} q_{1} d\right)$.

Putting these in the expression (85) for the reflected field and using the expression (B.8) for the dipole field, the total electric field (84) can be expressed in the following form, valid throughout the region above the metal:

$$
\begin{aligned}
E & =-\frac{1}{\pi \epsilon_{1}} \int \mathrm{d} p \mathrm{e}^{\mathrm{i} p \cdot \rho}\left[\delta(z-d) \hat{z} \cdot \mu \hat{z}+\frac{\mathrm{i}}{2 q_{1}}\left\{k_{1} \times\left(k_{1} \times \mu\right) \exp \left(\mathrm{i} q_{1}|z-d|\right)\right.\right. \\
& \left.\left.-\left[r_{12}^{\mathrm{P}}\left(p \hat{z}+q_{1} \hat{p}\right) \cdot \mu\left(p \hat{z}-q_{1} \hat{p}\right)+r_{12}^{\mathrm{S}} k_{1}^{2} \hat{z} \times \hat{p} \cdot \mu \hat{z} \times \hat{p}\right] \exp \left[\mathrm{i} q_{1}(z+d)\right]\right\}\right] .
\end{aligned}
$$

Here $\boldsymbol{k}_{1}$ is given by (B.9).

The field to be inserted in (81) is the total field evaluated at the dipole. The expression (88) for this field is singular at the dipole. A rule of thumb, which gives the correct result, is to interpret the field in (81) as the average of the field (88) just above and just below the dipole [34]. In fact it is not difficult to show that the singular terms give a real contribution which disappears when the imaginary part is formed in (81). With this interpretation we find the power dissipated to be

$$
\begin{aligned}
& P_{\text {tot }}=\frac{\omega}{2 \epsilon_{1}} \operatorname{Re} \int_{0}^{\infty} \mathrm{d} p \frac{p}{q_{1}}\left\{\left|\mu_{\perp}\right|^{2} p^{2}\left[1+r_{12}^{\mathrm{P}} \exp \left(2 \mathrm{i} q_{1} d\right)\right]\right. \\
& \left.\quad+\frac{1}{2}\left|\mu_{\|}\right|^{2}\left\{k_{1}^{2}\left[1+r_{12}^{\mathrm{S}} \exp \left(2 \mathrm{i} q_{1} d\right)\right]+q_{1}^{2}\left[1-r_{12}^{\mathrm{P}} \exp \left(2 \mathrm{i} q_{1} d\right)\right]\right\}\right\},
\end{aligned}
$$

where we have performed the integral over directions of $\boldsymbol{p}$. This formula is the basis for our discussion of the emission by a point dipole above a smooth surface.

As a preliminary to discussing the expression (89), we use it to obtain the power emitted by a free dipole in the absence of the surface. Setting the reflection coeffi- 
cients equal to zero we see that there is a contribution only from the range $0<p<$ $k_{1}$; the integrals are elementary and the result is

$P_{\text {free }}=\left(\epsilon_{1}^{1 / 2} \omega^{4} / 3 c^{3}\right)|\mu|^{2}$,

which is just the classical dipole radiation formula.

The integral in (89) may be interpreted as the integral of a $p$-dependent powerdissipated spectrum,

$P_{\text {tot }}=\int_{0}^{\infty} \mathrm{d} p \frac{\mathrm{d} P}{\mathrm{~d} p}$.

In fig. $S$ the solid curve shows $\mathrm{d} / \mathrm{d} p$, normalized to $P_{\mathrm{fr}}$ ee, as a function of $p$ for the case of a dipole placed at the surface $(d=0)$ and oriented perpendicular to the surface. The medium above the metal has been taken to be vacuum so $\epsilon_{1}=1$. We distinguish three distinct parts of this spectrum: (1) A rising spectrum in the range $0<p<\omega / c$, which is in fact not much different from the corresponding spectrum for a free dipole and which is associated with emission into photons above the surface. (2) A sharp peak at $p \gtrsim \omega / c$ associated with surface plasmon emission. (3) A broad peak occurring at $p \gg \omega / c$ which is associated with electron-hole excita-

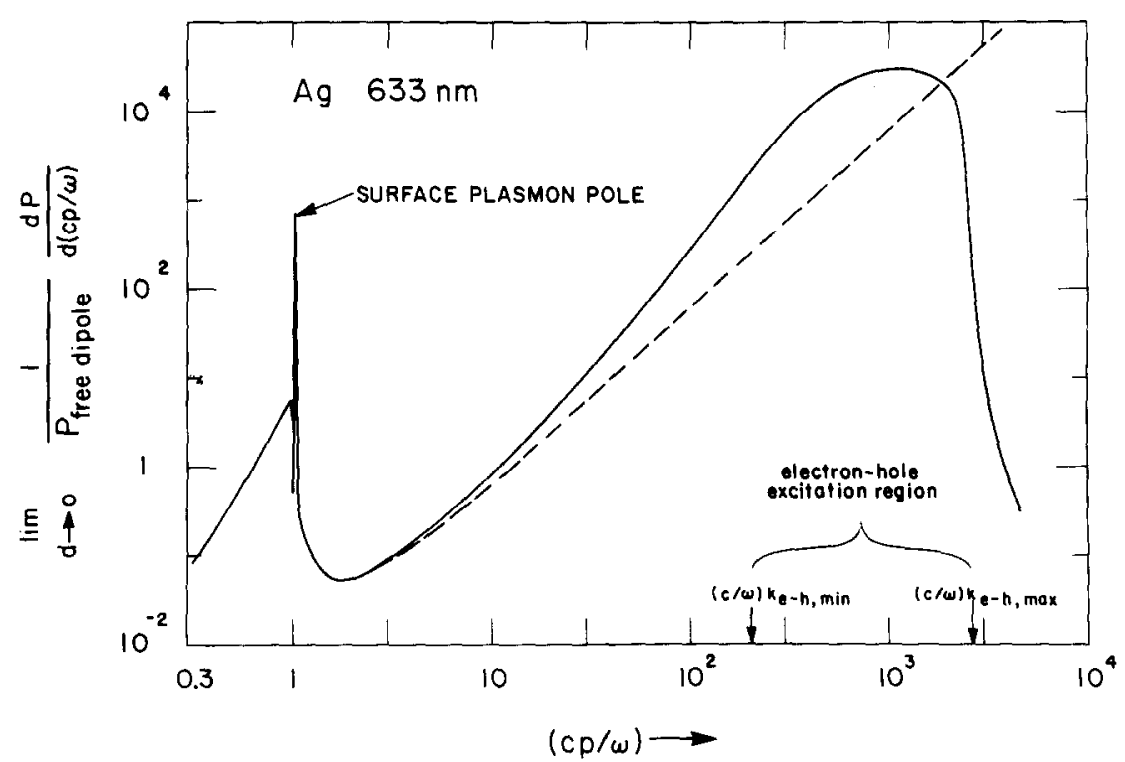

Fig. 5. The $p$-dependent power dissipated spectrum defined in eq. (91) for an oscillating point dipole placed on and oriented normal to a $\mathrm{Ag}$ surface. The solid curve corresponds to our nonlocal model, the dashed curve to the local result. The two models are identical for small values of $p$. 
tions. The total power dissipated may therefore be written

$P_{\text {tot }}=P_{\text {photon }}+P_{\mathrm{SP}}+P_{\mathrm{e}-\mathrm{h}}$.

In table 1 we show each of these contributions, i.e., the area under the corresponding part in fig. 5 , for three commonly used laser frequencies in the visible. The dipole is placed $1.5 \AA$ from the metal and oriented perpendicular to the surface.

The dashed curve in fig. 5 is obtained with a local $\epsilon_{2}(\omega)$ representing the same optical data. The rough agreement between the two curves in the high- $p$ region is misleading, since the dissipation in the local theory is proportional to the electron scattering rate while that in the nonlocal theory is determined by electron-hole excitation and is independent of that rate.

The features of the power-dissipated spectrum shown in fig. 5 can be understood in terms of the dispersion relations for bulk modes in the metal, shown in fig. $6 \mathrm{a}$, and for modes associated with the metal-vacuum interface, shown in fig. $6 \mathrm{~b}$. The propagating modes in the bulk metal are the transverse photon mode and the longitudinal plasmon mode, both occurring for $\omega>\omega_{p}$ which is well above the range of frequencies of interest in our discussion. The other feature of the bulk mode spectrum is the broad continuum of electron-hole excitations, which occur at any given frequency for values of the three-dimensional propagation vector $k$ in the range [35]:

$\left(2 m \omega / \hbar+k_{\mathrm{F}}^{2}\right)^{1 / 2}-k_{\mathrm{F}}<k<\left(2 m \omega / \hbar+k_{\mathrm{F}}^{2}\right)^{1 / 2}$.

The modes associated with the interface are shown in fig. $6 \mathrm{~b}$ where the abscissa is the component of the propagation vector parallel to the interface. The photon modes above the metal form a continuum $0<p<\omega / c$ coming from the projection of the light line, $\omega=c k$, onto the interface. The continuum of electron-hole excitations is projected into a still broader continuum, $0<p<\left(2 m \omega / \hbar+k_{\mathrm{F}}^{2}\right)^{1 / 2}+k_{\mathrm{F}}$, with the same upper edge as the bulk excitations. The new feature is the surface plasmon mode, a discrete surface mode with dispersion relation [36]:

$p=p_{\mathrm{SP}} \equiv\left(\frac{\epsilon_{1} \epsilon_{2}}{\epsilon_{1}+\epsilon_{2}}\right)^{1 / 2} \frac{\omega}{c}, \quad \epsilon_{2}<-\epsilon_{1}<0$.

Table 1

Computed results for a point dipole $1.5 \mathrm{~A}$ from and perpendicular to a $\mathrm{Ag}$ surface; the parameters are $\epsilon_{1}=1, \epsilon_{\mathrm{b}}=3.6, \hbar \omega_{\mathrm{p}}=9.33 \mathrm{eV}, \hbar \nu=0.058 \mathrm{eV}, v_{\mathrm{F}}=1.4 \times 10^{8} \mathrm{~cm} / \mathrm{s}$, and $k_{\mathrm{F}}=1.2 \mathrm{X}$ $10^{8} \mathrm{~cm}^{-1}$; the powers are all normalized with respect to the power emitted by a free dipole in medium 1

\begin{tabular}{lllll}
\hline$\hbar \omega(\mathrm{eV})$ & $\epsilon_{2}(\omega)$ & $P_{\text {photon }}$ & $P_{\mathrm{SP}}$ & $P_{\mathrm{e}-\mathrm{h}}$ \\
\hline 1.96 & $-19.06+0.67 \mathrm{i}$ & 1.24 & 2.34 & $2.63 \times 10^{6}$ \\
2.41 & $-11.38+0.36 \mathrm{i}$ & 1.07 & 3.24 & $1.71 \times 10^{6}$ \\
2.60 & $-9.27+0.29 \mathrm{i}$ & 1.00 & 3.72 & $1.47 \times 10^{6}$ \\
\hline
\end{tabular}



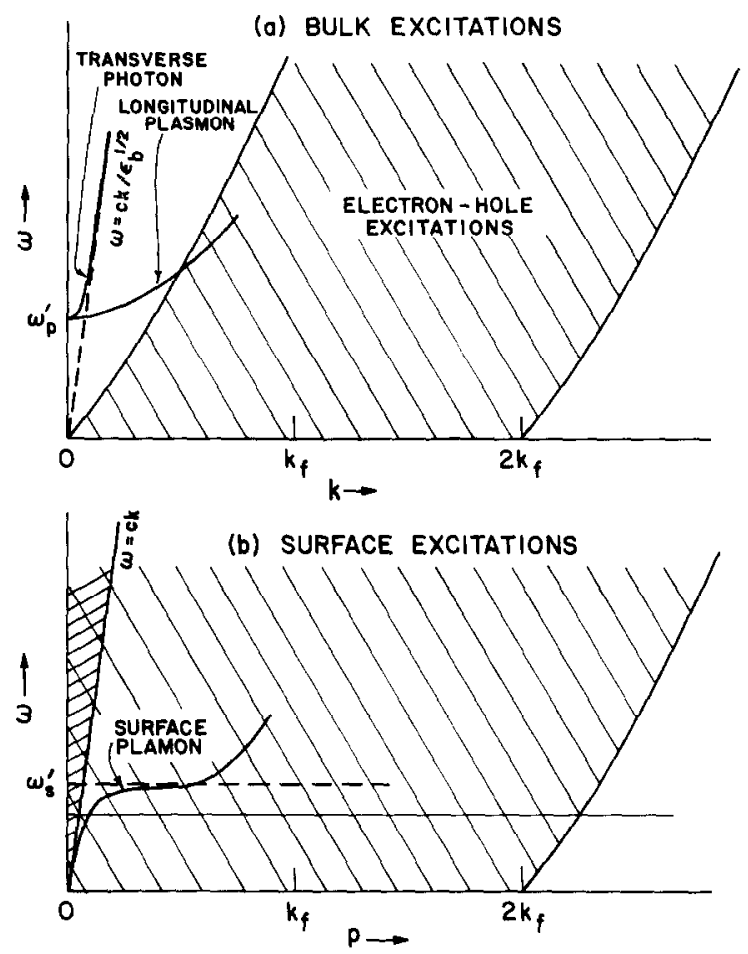

Fig. 6. Schematic excitation spectrum of a free-electron metal. In (a), $\omega_{p}^{\prime}=\epsilon_{b}^{-1 / 2} \omega_{p}$ is the bulk plasmon frequency. In (b), $\omega_{\mathrm{s}}^{\prime}=\left(\epsilon_{\mathrm{b}}+1\right)^{-1 / 2} \omega_{\mathrm{p}}$ is the asymptotic surface plasmon frequency for a local metal. The medium above the metal is vacuum. The thin horizontal line in (b) corresponds to the abscissa in fig. 5.

The projections of the bulk plasmon and transverse photon in the metal are not shown.

The horizontal line in fig. $6 \mathrm{~b}$ corresponds to the frequency $\omega$ used in fig. 5. The photon emission corresponds to the range $0<p<\omega / c$, to the left of the light line. The surface plasmon emission corresponds to the point on the surface plasmon dispersion curve just to the right of the light line. Finally there is the broad underlying continuum of electron-hole excitations. Phase space factors strongly. weight the large $p$ portions of the continua, leading to peaks near the upper end of the ranges.

The above qualitative description has been in terms of an ideal electron gas model with infinite relaxation time. The spectrum in fig. 5 and the results in table 1 have been calculated with an electron scattering rate $v$ appropriate for $\mathrm{Ag}$. The effects of electron scattering are not large. If $\nu$ were reduced to zero, the principal effects would be a narrowing of the surface plasmon peak and a decrease in $\mathrm{d} P / \mathrm{d} p$ in the range $p_{\mathrm{SP}} \leqslant \mathrm{p}<<k_{\mathrm{F}}$, above the surface plasmon peak but well below 
the electron-hole peak. The results in table 1 would be changed very little. If, on the other hand, $\nu$ were increased there would be correspondingly little change until the increase exceeded an order of magnitude. At this point, the power dissipated by the dipole would begin to have a substantial electron scattering contribution. Such large scattering rates would have the additional effect of reducing the peak gains shown in figs. 3 and 4 . A liquid metal has scattering rates in this range ( $h v \approx 1.4$ $\mathrm{eV}$ for $\mathrm{Hg}$ ) and so should exhibit a significantly reduced image enhancement gain.

From the above discussion, we see that the photon emission and surface plasmon emission, since they occur at values of $p \ll k_{\mathrm{F}}$, can be described in the local approximation, in which the reflection coefficients are given by (77). For $0<p<$ $\omega / c$ we see that $q_{1}$ is real and $q_{2}$ is pure imaginary, corresponding to photon emission, i.e., propagating modes above the metal and evanescent waves in the metal. The surface plasmon emission corresponds to a pole in the P-polarized reflection coefficient $r_{12}^{\mathrm{P}}$ which occurs at $p=p_{\mathrm{SP}}$, given by (94). Here both $q_{1}$ and $q_{2}$ have large positive imaginary parts, corresponding to evanescent waves confined to the surface. In the neighborhood of the pole

$r_{12}^{\mathrm{P}} \approx-\frac{2 \epsilon_{1} \epsilon_{2}}{\epsilon_{2}^{2}-\epsilon_{1}^{2}} \frac{p_{\mathrm{SP}}}{p-p_{\mathrm{SP}}}$,

and, since $\operatorname{Im}\left\{p_{\mathrm{SP}}\right\}>0$, the surface plasmon contribution to the power dissipated is $i \pi$ times the residue of the integrand of (89) at $p=p_{\mathrm{Sp}}$. After some rearrangement we find

$P_{\mathrm{SP}}=\frac{\pi c^{2}}{\omega} \operatorname{Re}\left\{\frac{\left(-\epsilon_{2}\right)^{1 / 2} p_{\mathrm{SP}}^{5}}{\epsilon_{1}^{3 / 2}\left(\epsilon_{1}-\epsilon_{2}\right)}\left(\left|\mu_{1}\right|^{2}-\frac{\epsilon_{1}}{2 \epsilon_{2}}\left|\mu_{\|}\right|^{2}\right) \exp \left[-2\left(-\epsilon_{1} / \epsilon_{2}\right)^{1 / 2} p_{\mathrm{SP}} d\right]\right\}$

which is a result usually expressed in terms of the decay rate into surface plasmons of a fluorescing molecule near a metal $[16,37,38]$. The results for $P_{\mathrm{SP}}$ given in table 1 were obtained by numerical integration of (89) over the symmetrical range $\omega / c<p<2 p_{\mathrm{SP}}-\omega / c$. They agree within a few percent with those given by (96).

The broad electron-hole excitation peak, shown in fig. 5 , occurs for $\omega / c<<$ $p \leq 2 k_{k}$, a range of $p$ for which the quasistatic approximation is appropriate, i.e., the P-polarized reflection coefficient is given by (43) and the S-polarized reflection coefficient vanishes. We can thus approximate $P_{\mathrm{e}-\mathrm{h}}$ by

$P_{\mathrm{e}-\mathrm{h}}=\frac{\omega}{2 \epsilon_{1}} \int_{0}^{\infty} \mathrm{d} p p^{2}\left(\left|\mu_{1}\right|^{2}+\frac{1}{2}\left|\mu_{\|}\right|^{2}\right) \mathrm{e}^{-2 p d} \operatorname{Im}\left(r_{12}^{\mathrm{P}}\right)$,

in which we use the quasistatic result (43) for $r_{12}^{\mathrm{P}}$. The dissipation in this peak is not associated with any pole or with the emission of any normal mode in either medium, rather it is associated with what we term driven surface modes, coupled motions of the electric field and the electron plasma which are present only when there is a driving field. The dissipation is through excitation of electron-hole 
pairs. The results for $P_{\mathrm{e}-\mathrm{h}}$ given in table 1 were obtained by numerical integration of (89) over the range $2 p_{\mathrm{SP}}-\omega / c<p \leqslant \infty$ and agree well with the approximate formula (97).

The distance dependence of these results can be understood qualitatively from our approximate formulas and fig. 5. Consider first the expression (97) for $P_{\mathrm{e}-\mathrm{h}}$. The integrand for $d=0$ has a broad peak with maximum at $p \approx 2 k_{\Gamma}$. The exponential factor in the integrand will cut this peak off at $p \approx d^{-1}$, so $P_{\mathrm{e}-\mathrm{h}}$ will diminish rapidly as $d$ increases. When $c / \omega \gg d \gg\left(k_{\mathrm{F}}\right)^{-1}$, corresponding to $d \approx 50-500 \Omega$ for the calculations shown in fig. 5 , the local approximation can be used in (43) and the expression (97) for the power can be evaluated explicitly:

$P \approx \frac{\omega}{8 \epsilon_{1} d^{3}}\left(\left|\mu_{\perp}\right|^{2}+\frac{1}{2}\left|\mu_{\|}\right|^{2}\right) \operatorname{Im} \frac{\epsilon_{2}-\epsilon_{1}}{\epsilon_{2}+\epsilon_{1}}$.

Here the power is strictly speaking the dissipation due to electron scattering, not electron-hole excitations. In this range the power (98) is in fact negligible compared with $P_{\mathrm{SP}}$ which, as we see from (96), does not begin to diminish until $d \approx p_{\mathrm{SP}}^{-1}\left(-\epsilon_{2} / \epsilon_{1}\right)^{1 / 2}$, which corresponds to $d \approx 10^{3} \AA$ for the parameters used in fig. 5 . For still larger $d, P_{\mathrm{SP}}$ diminishes exponentially, becoming negligible compared with $P_{\text {photon }}$ when $d \gg c / \omega$.

In applying the results of this section to molecular fluorescence we need only make the identification:

$\gamma_{\text {tot }}=P_{\text {tot }} / \hbar \omega$,

where $\gamma_{\text {tot }}$ is the total decay rate and $P_{\text {tot }}$ is the total power dissipated given by (89) in which $\mu$ is taken to be twice the transition matrix element of the dipole operator. In the same way the partial decay rates are related to the corresponding partial power dissipations as in (92),

$\gamma_{\text {tot }}=\gamma_{\text {photon }}+\gamma_{\mathrm{SP}}+\gamma_{\mathrm{e}-\mathrm{h}}$.

\section{Conclusion}

We have here discussed two aspects of the electromagnetic interaction of a molecule with a metal surface, the image enhancement effect and the power dissipation. Both have important applications other than to Raman scattering. For example, the solution we have obtained for the image enhancement problem can be applied to the problem of determining shifts in the vibration frequencies of adsorbed molecules as well as to the problem of the Van der Waals forces on a molecule near a surface where finite size is important.

In conclusion we wish to emphasize that, although our calculations are based on rather idealized models of the molecule and the metal, the electromagnetic effects we have discussed are surely present. The final explanation of the SERS 
effect may involve other ingredients but it cannot ignore these basic electromagnetic effects.

\section{Appendix A}

Here we obtain the formulas (8) and (9) using known formulas from standard references. We begin by writing

$\mathrm{e}^{\mathrm{i} p \cdot \rho-p z}=\mathrm{e}^{-p d} \exp \left(\mathrm{i} p \cdot r_{1}-p \hat{z} \cdot r_{1}\right)=\sum_{l=0}^{\infty} \frac{\mathrm{e}^{-p d}\left(-p r_{1}\right)^{l}}{l !}\left(\hat{z} \cdot f_{1}-\mathrm{i} \hat{p} \cdot \hat{r}_{1}\right)^{l}$

But

$\hat{z} \cdot \hat{r}_{1}=\cos \theta_{1} \quad$ and $\hat{p} \cdot \hat{r}_{1}=\sin \theta_{1} \cos \left(\phi_{1}-\phi\right)$,

where $\phi_{1}$ is the azimuthal angle of $\dot{r}_{1}$ and $\phi$ that of $\hat{p}$. Using the formula (ref. [21], p. 55)

$(\cos \theta+\mathrm{i} \sin \theta \cos \phi)^{l}=P_{l}(\cos \theta)+2 \sum_{m=1}^{l}(-\mathrm{i})^{m} \frac{l !}{(l+m) !} P_{l}^{m}(\cos \theta) \cos m \phi$,

and the relation [39]

$P_{l}^{-m}(\cos \theta)=(-1)^{m} \frac{(l-m) !}{(l+m) !} P_{l}^{m}(\cos \theta)$,

we can write

$\left(\hat{z} \cdot \hat{r}_{1}-\mathrm{i} \hat{p} \cdot \hat{r}_{1}\right)^{l}=\sum_{m=-l}^{l} \mathrm{i}^{m} \frac{l !}{(l+m) !} P_{l}^{m}\left(\cos \theta_{1}\right) \exp \left[\mathrm{im}\left(\phi_{1}-\phi\right)\right]$.

Inserting this in (A.1) gives the desired formula (14).

To obtain (15) we begin with the formula (ref. [27], p. 68)

$P_{l}^{-m}(\cos \theta)=\frac{1}{(l+m) !} \int_{0}^{\infty} \mathrm{d} t t^{l} \mathrm{e}^{-t \cos \theta} J_{m}(t \sin \theta), \quad 0<\theta<\pi / 2$.

Using (A.4) and the relation (ref. [27], p. 23)

$P_{l}^{m}(-x)=(-1)^{l+m} P_{l}^{m}(x)$,

we can then write

$P_{l}^{m}\left(\cos \theta_{1}\right)=\frac{(-1)^{l}}{(l-m) !} \int_{0}^{\infty} \mathrm{d} t t^{l} \exp \left(t \cos \theta_{1}\right) J_{m}\left(t \sin \theta_{1}\right), \quad \pi / 2<\theta_{1}<\pi$. 
Now (ref. [27], p. 26)

$J_{m}(z)=\frac{(-\mathrm{i})^{m}}{(2 \pi)} \int_{0}^{2 \pi} \mathrm{d} \phi \mathrm{e}^{\mathrm{i} z \cos \phi} \mathrm{e}^{\mathrm{i} m \phi}$.

Putting this in (A.8) and replacing $t \rightarrow p r_{1}, \phi \rightarrow \phi-\phi_{1}$, we get.

$r_{1}^{-l-1} P_{l}^{m}\left(\cos \theta_{1}\right) \mathrm{e}^{\mathrm{i} m \phi_{1}}=\frac{(-\mathrm{i})^{m}(-1)^{l}}{2 \pi(l-m) !} \int_{0}^{\infty} \mathrm{d} p \int_{0}^{2 \pi} \mathrm{d} \phi p^{l} \mathrm{e}^{\mathrm{i} m \phi}$

$\times \exp \left\{\mathrm{ip} r_{1} \sin \theta_{1} \cos \left(\phi-\phi_{1}\right)+p r_{1} \cos \theta_{1}\right\}$.

But

$p r_{1} \sin \theta_{1} \cos \left(\phi-\phi_{1}\right)=\boldsymbol{p} \cdot \rho, \quad r_{1} \cos \theta=z-d$.

Hence, writing $\mathrm{d} \boldsymbol{p}=p \mathrm{~d} p \mathrm{~d} \phi$, we get the formula (15).

\section{Appendix B. The fields for a point dipole}

Here we calculate expressions for the fields due to a point dipole $\mu \mathrm{e}^{-\mathrm{i} \omega t}$ in an infinite medium of dielectric constant $\epsilon_{1}$. The dipole is placed on the $z$-axis a distance $d$ above the $x y$-plane. We introduce a Fourier expansion of the electric and magnetic fields:

$E(r)=\int \mathrm{d} k \mathrm{e}^{\mathrm{i} k \cdot r} E_{k}, B(r)=\int \mathrm{d} \boldsymbol{k} \mathrm{e}^{\mathrm{i} k \cdot r} B_{k}$.

This is in the spirit of the Weyl approach to the Sommerfeld problem [40]. The Maxwell equations (82) and (83) become

$B_{k}=\frac{c}{\omega} k \times E_{k}$

$\boldsymbol{k} \times \boldsymbol{B}_{k}+\epsilon_{1} \frac{\omega}{c} E_{k}=-\frac{\omega}{2 \pi^{2} c} \mu \exp \left(-\mathrm{i} k_{z} d\right)$.

The solution of these equations is

$E_{k}=-\frac{1}{2 \pi^{2} \epsilon_{1}}\left[\mu+\frac{k \times(k \times \mu)}{k^{2}-k_{1}^{2}}\right] \exp \left(-\mathrm{i} k_{z} d\right)$,

$\boldsymbol{B}_{k}=\frac{\omega}{2 \pi^{2} c} \frac{k \times \mu}{k^{2}-k_{1}^{2}} \exp \left(-\mathrm{i} k_{z} d\right)$,

where

$k_{1}^{2}=\epsilon_{1} \omega^{2} / c$. 
The fields are given by inserting these expressions in the integrals (B1). If we introduce cylindrical coordinates,

$\boldsymbol{k}=\boldsymbol{p}+k_{z} \hat{z}, \quad r=\rho+z \hat{z}$,

these integrals become

$\boldsymbol{E}(\boldsymbol{r})=-\frac{1}{2 \pi^{2} \epsilon_{1}} \int \mathrm{d} \boldsymbol{p} \mathrm{e}^{\mathrm{i} p \cdot \rho} \int_{\infty}^{\infty} \mathrm{d} k_{z} \exp \left[\mathrm{i} k_{z}(z-d)\right]\left[\mu+\frac{\boldsymbol{k} \times(\boldsymbol{k} \times \mu)}{k_{z}^{2}-q_{1}^{2}}\right]$,

and a similar expression for $\boldsymbol{B}(\boldsymbol{r})$. Here

$q_{1}=\left(k_{1}^{2}-p^{2}\right)^{1 / 2}, \quad \operatorname{Im}\left\{q_{1}\right\}>0$.

For $z>d$ we perform the $k_{z}$-integral by completing the contour in the upper halfplane; the integral is then $2 \pi \mathrm{i}$ times the residue at the pole at $k_{z}=q_{1}$. For $z<d$ we complete in the lower half-plane and the integral is $-2 \pi i$ times the residue at $k_{z}=$ $-q_{1}$. In addition there is a contribution to the integral equal to $2 \pi \delta(z-d)$ times the limit as $\left|k_{z}\right| \rightarrow \infty$ of the quantity in square brackets in the integrand. The result is summarized in the formula

$E(r)=-\frac{1}{\pi \epsilon_{1}} \int \mathrm{d} p \mathrm{e}^{\mathrm{i} p \cdot p}\left[\delta(z-d) \hat{z} \cdot \mu \hat{z}+\frac{\mathrm{i}}{2 q_{1}} k_{1} \times\left(k_{1} \times \mu\right) \exp \left(\mathrm{i} q_{1}|z-d|\right)\right]$,

where

$k_{1}=p+q_{1} \frac{z-d}{|z-d|} \hat{z}$.

The corresponding formula for the magnetic field is

$\boldsymbol{B}(\boldsymbol{r})=\frac{\mathrm{i} \omega}{2 \pi c} \int \mathrm{d} \boldsymbol{p} \mathrm{e}^{\mathrm{i} p \cdot \rho} \frac{\boldsymbol{k}_{1} \times \mu}{q_{1}} \exp \left(\mathrm{i} q_{1}|z-d|\right)$.

These formulas express the dipolar fields as a superposition of plane waves with complex propagation vector $k_{1}$. When $p<k_{1}=\epsilon_{1}^{1 / 2} \omega / c$, the propagation vector is real, corresponding to radiated plane waves. When $p>k_{1}$, the $z$-component of the propagation vector is imaginary, corresponding to the near fields of the dipole.

\section{References}

[1] R.P. Van Duyne, in Chemical and Biological Applications of Lasers, Vol. 4, Ed. C.B. Moore (Academic Press, 1979) ch. 5, and references therein.

[2] R.R. Chance, A. Prock and R. Silbey, in: Advances in Chemical Physics, Vol. 37, Eds. I. Prigogine and S.A. Rice (Wiley, New York, 1978) p. 1. 
[3] S. Andersson and B.N.J. Persson, Phys. Rev. Letters 45 (1980) 1421;

G.D. Mahan and A.A. Lucas, J. Chem. Phys. 68 (1978) 1344.

[4] P. Hollins and J. Pritchard, Chem. Phys. Letters 75 (1980) 378.

[5] A. Hartstein, J.R. Kirtley and J.C. Tsang, Phys. Rev. Letters 45 (1980) 201.

[6] E.M. Lifshitz, Zh. Eksperim. i. Teor. Fiz. 29 (1955) 94 [Engl. Transl. Soviet Phys.-JETP 2 (1956) 73];

I.E. Dzyaloshinskii, E.M. I ifshitz and L.P. Pitaevskii, Advan. Phys. 10 (1961) 165 .

[7] R. Naaman, S.J. Buelow, O. Cheshnovsky and D.R. Herschbach, J. Phys. Chem. 84 (1980) 2692.

[8] A. Otto, J. Timper, J. Billman and I. Pockrand, Phys. Rev. Letters 45 (1980) 46.

[9] B.H. Loo and T.E. Furtak, Chem. Phys. Letters 71 (1980) 68.

[10] J.E. Rowe, C.V. Shank, D.A. Zwemer and C.A. Murray, Phys. Rev. Letters 44 (1980) 1770

D.A. Zwemer, C.V. Shank and J.E. Rowe, Chem. Phys. Letters 73 (1980) 201.

[11] P.N. Sanda, J.M. Warlaumont, J.E. Demuth, J.C. Tsang, K. Christmann and J.A. Bradley, Phys. Rev. Letters 45 (1980) 1519.

[12] F.W. King, R.P. Van Duyne and G.C. Schatz. J. Chem. Phys. 69 (1978) 4472;

G.C. Schatz and R.P. Van Duyne, Surface Sci. 101 (1980) 425.

[13] S. Efrima and H. Metiu, J, Chem. Phys. 70 (1979) 1602, 2297; Surface Sci. 92 (1980) 417.

[14] W.H. Weber and G.W. Ford, Phys. Rev. Letters 44 (1980) 1774.

[15] G. Korzeniewski, T. Maniv and H. Metiu, Chem. Phys. Letters 73 (1980) 212; J. Chem. Phys., to be published.

[16] W.H. Weber and C.F. Eagen, Opt. Letters 4 (1979) 236.

[17] C.F, Eagen, W.H. Weber, S.L. McCarthy and R.W. Terhune, Chem. Phys. Letters 75, 274 (1980).

[18] F. Garcia-Moliner and F. Flores, Introduction to the theory of Solid Surfaces (Cambridge Univ. Press, Cambridge, 1979).

[19] H. Faxén, Ann. Physik 68 (1922) 89.

[20] D.K. Ross, Australian J. Phys. 21 (1968) 817.

[21] F.B. Hildebrand, Methods of Applied Mathematics, 2nd ed. (Prentice-Hall, Englewood Cliffs, NJ, 1962) p. 10.

[22] K.L. Kliewer and R. Fuchs, Phys. Rev. 172 (1968) 607;

R. Fuchs and K.L. Kliewer, Phys. Rev. 185 (1969) 905.

[23] N.D. Mermin, Phys. Rev. B1 (1970) 2362;

K.L. Kliewer and R. Fuchs, Phys. Rev. 181 (1969) 552.

[24] N.E. Christensen, Phys. Status Solidi (b) 54 (1972) 551.

[25] E.U. Condon and G.H. Shortley, Theory of Atomic Spectra (Cambridge Univ. Press, Cambridge, 1957) p. 106.

[26] C.A. Coulson, Proc. Roy. Soc. Edinburgh 61A (1941) 20.

[27] W. Magnus and F. Oberhettinger, Formulas and Theorems for the Special Functions of Mathematical Physics (Chelsea, New York, 1949) pp. 51, 55.

[28] M. Abramowitz and I.A. Stegun, Handbook of Mathematical Functions (US Government Printing Office, Washington, DC, 1964) p. 437.

[29] M. Otter, Z. Physik 161 (1961) 163;

M.M. Dujardin and M.L. Theye, J. Phys. Chem. Solids 32 (1971) 2033;

W.H. Weber and S.L. McCarthy, Phys. Rev. B12 (1975) 5643.

[30] I.L. Fabelinskii, Molecular Scattering of Light (Plenum, New York, 1968) pp. 555-560.

[31] P.K. Antoniewicz, J. Chem. Phys. 56 (1972) 1711;

W.C. Meixner and P.R. Antoniewicz, Phys. Rev. B13 (1976) 3276.

[32] A. Sommerfeld, Partial Differential Equations in Physics (Academic Press, New York, 1949) ch. VI. 
[33] B.N.J. Persson, J. Phys. C11 (1978) 4251.

[34] J. Schwinger, Phys. Rev. 75 (1949) 1912.

[35] D. Pines, Elementary Excitations in Solids (Benjamin, New York, 1964) p. 149.

[36] H. Raether, in: Physics of Thin Films, Vol. 9, Eds. G. Hass, M.H. Francombe and K.W. Hoffmann (Academic Press, New York, 1977) p. 145.

[37] M.R. Philpott, J. Chem. Phys. 62 (1975) 1812;

H. Morawitz and M.R. Philpott, Phys. Rev. B10 (1974) 4863.

[38] G.S. Agarwal and H.D. Vollmer, Phys. Status Solidi (b) 79 (1977) 249.

[39] A.R. Edmonds, Angular Momentum in Quantum Mechanics (Princeton Univ. Press, Princeton, NJ, 1957) p. 23.

[40] H. Weyl, Ann. Physik 60 (1919) 481. 